\title{
Fifty Shades of Ultrastructural Pathology in One Thousand Sural Nerves
}

\author{
Juan M Bilbao, MD, FRCP, University of Toronto
}

Over a span of forty years a single pathologist was involved in the study of peripheral nerve biopsies at the University of Toronto Hospitals. Every specimen (mostly sural nerve) was processed for electron microscopy. I will describe the challenges posed by the artifacts identified by transmission electron microscopy (TEM), caused by rough handling of tissue, the use of improper fixatives, and the adaptation (notwithstanding its shortcomings) of a fixative of low osmolality tailored for nerve tissue. I will illustrate the salient features in the many cases where a specific diagnosis was achieved based on TEM. The universal configuration of overlapping perineurial cells joined by an abundant number of zonula occludens confirms that the perineurium regulates the endoneurial milieu and that it is a bidirectional barrier to the intrusion of dyes. The endoneurial vessels consistently demonstrate tight junctions between endothelial cells and a complementary outer pericyte or smooth muscle cell, thus different from the capillaries of striated muscle. The ultrastructural demonstration of tubulo-reticular (undulating tubules) inclusions in endothelium is essential in the diagnosis of endoneurial microvasculitis that occurs in patients suffering from systemic lupus erythematosus and related diseases, and AIDS. The sural nerve with an endoneurial surface area of 0.6 to $1.3 \mathrm{~mm}$ contains about 7,000 myelinated fibers (MF), and 25,000 unmyelinated fibers per square centimetre. In patients suspected of having inflammatory neuropathy, the detection of macrophage-mediated myelin stripping is important because it narrows the differential diagnosis to three entities: Guillain-Barré syndrome, chronic inflammatory demyelinating polyneuropathy, and IgM paraproteinemic polyneuropathy. Viewed under the electron microscope, tongues of invading macrophages penetrate the Schwann cell (SC) basal lamina and cell membrane, enter into the intraperiod line of myelin and strip and engulf myelin lamellae resulting in segmental demyelination. In addition, in chronic disease, a disruption of the blood-nerve barrier occurs because of fenestration of the endothelium.

Amyloid deposition in nerve resulting in neuropathy can be divided into hereditary (transthyretin mutations) and acquired (light chain) forms. TEM evinces the characteristic "rigid" amyloid fibrils in endoneurium, in between perineurial leaves, and percolating into vessels. Difficulties arise when one overlooks the fact that the oxytalan fibril that inhabits endoneurium is an impersonator of amyloid. Peripheral nerves are affected in all types of leprosy. Because patients with lepromatous leprosy lack cellmediated immunity to the organism, M. leprae manifests unchecked growth in nerves. Bacilli are found in foamy macrophages (Lepra cells) forming globi, myelinated Schwann cells, perineurial cells, incorporated into axons, and in endothelial cells. The latter finding is supportive of the hypothesis that bacilli gain access to nerves through haematogenous spread and colonization of endothelium. Neuropathy develops in $58 \%$ of patients with paraproteinemia, particularly IgM. The detection of widely spaced myelin (WSM) prefigures the presence of a subset of IgM paraproteins with activity against MAG (myelin-associated glycoprotein), while uncompacted myelin (UCM) is associated with the POEMS syndrome (polyneuropathy, organomegaly, endocrinopathy, M-protein, skin abnormalities). Lack of apposition of the outer (extracellular) aspects of the SC membrane typifies WSM. The intraperiod line that normally consists of a 2-4 nm separation is increased to $20-30 \mathrm{~nm}$ and represents a virtual extracellular space. The intrusion of anti- MAG antibodies that opens the intraperiod line is unveiled with immunogold anti-Mag preparations. In UCM the major dense line fails to form because the inner (cytoplasmic) aspects of the SC membrane do not fuse. In monoclonal immunoglobulin deposition disease, there are coalescent deposits of amorphous material in endoneurium (Congo-red negative, consisting of single light chain, truncated isolated heavy chain or the entire immunoglobulin molecule) 
By TEM they appear granular, lacking the distinctive appearance of amyloid (strait fibrils composed of two parallel subunits). I will illustrate a case where anti-kappa immunogold stain highlights the powdery material.

In many of the polyneuropathies associated with sphigolipidosis, the SCs harbour pathognomonic inclusions. In Krabbe disease there are prismatic, curved or straight, some optically empty shard-like inclusions that are also observed in endoneurial macrophages. In metachromatic leukodystrophy accumulation of sphingolipid sulphatide leads to segmental demyelination and the formation of "tuffstone" cytoplasmic inclusions, and unusual lysosomes with prismatic and herringbone patterned contents. By contrast, in Fabry disease vast numbers of lipid inclusions arise in perineurial cells, endothelium, and vascular smooth muscle, but not in SCs. Inclusions ("banana bodies") that are electron lucent membrane-bound clefts up to $5 \mu \mathrm{m}$ in diameter with rectangular or angulated profiles, occur in the cytoplasm of myelinated SCs in Farber disease. Curved stacks of lamellae and fingerprint cytoplasmic profiles portend neuronal ceroid lipofuscinosis. Bilaminar or multilaminar cytosomes and lipid with a fusiform configuration, detected in the cytoplasm of SCs, characterize peroxisomal disorders (adrenomyeloneuropathy). Lipid accumulation in the acquired lipidosis caused by the toxic effects of amphiphilic drugs (amiodarone, chloroquine) is notable in peripheral nerves. SCs, endothelium, perineurial cells, and smooth muscle cells contain membrane-bound osmiophilic material, lysosomes with a lamellar or granuoreticular profile, and aggregates of curvilinear bodies. In Adult Polyglucosan Body Disease, Glycogen brancher enzymel mutation results in the accumulation of amylopectin-like polysaccharide or polyglucosan bodies in many tissues and in peripheral nerves. These bodies, 10 to $70 \mathrm{um}$ in diameter, occur in myelinated axons and consist of randomly arranged 6-8 nm branched filaments. They are similar to corpora amylacea, Lafora bodies and Bielschowki bodies.

Charcot Marie Tooth (CMT) disease and cognate disorders are the most common monogenetically inherited diseases of the peripheral nervous system. Although TEM rarely leads to a specific diagnosis, ultrastructural examination helps define phenotypes. The identification of onion bulbs uniformly in all fascicles suggests CMT1 (duplication of peripheral myelin protein $22\{P M P 22\}$ ), pseudo-onion bulbs and regenerating clusters point to CMT2 (missense mutation of $M f n 2$ ), intermediate forms that combine demyelinating and axonal features exemplify the pathologies of X-linked CMT, and autosomal dominant intermediate CMT. The group of congenital myopathies/Dejerine-Sottas syndrome displays onion bulbs with hypomyelination and onion bulbs composed of rings of basal lamina. Abnormalities in the configuration of myelin with redundant loops delineate CMT4. Tomaculous formations are ubiquitous in PMP 22 deletions (hereditary neuropathy with pressure palsies). The tomacula is a doublefolded redundant myelin loop that wraps around the axon to varying degrees, forming up to 3 or 4 complete tightly packed turns ("jelly roll"). This occurs in up to $10 \%$ of axons viewed on cross orientation. Giant Axonal Neuropathy is an autosomal recessive disorder of intermediate filament organization. Nerve sections show massively dilated axons containing crowded filaments (lacking the sidearm typical of normal neurofilament) that swirl and stream in haphazard configurations. This is a systemic disease as whorls of filaments are also present in SCs and endothelium. Patients with another autosomal recessive degenerative disorder (termed Neuroaxonal Dystrophy) manifest motor-cognitive regression followed by neuropathy. Dystrophic axons, or axonal spheroids, incorporate aggregates of tubulovesicular profiles with clusters of mitochondria. Intramitochondrial crystaloids in SCs is a rare finding in the neuropathies associated with mitochondrial cytopathies.

Finally, I will show occasional non-specific inclusions detected on routine examination of nerves that mimic pathological change. These include paracrystaline inclusions in non-myelinating Schwann cells, intra-axonal aggregates of cytomembranes, intra-axonal clusters of paracrystaline bodies, and Reich Pi granules of myelinated SCs.

Ref: JM Bilbao \& R Schmidt. Biopsy Diagnosis of Peripheral Neuropathy. Springer; 2014. 